\title{
Cystic Papillary Thyroid Carcinoma: A Case Report
}

\author{
Darwin Totesora and Ma. Theresa Chua-Agcaoili \\ Section of Endocrinology, Diabetes and Metabolism, The Medical City, Pasig City, Philippines
}

\begin{abstract}
Cystic nodule is an unusual presentation of Papillary Thyroid Carcinoma (PTC), seen in less than $10 \%$ of cases. Even by ultrasound, finding a thyroid cyst carries a less than $5 \%$ chance of malignancy. We present a case of a 45 -year-old male, who came in for incidental finding of a palpable thyroid mass with no significant predisposing history. Thyroidectomy was done and immunohistochemical staining confirmed it to be papillary thyroid cancer. He underwent high dose radiation therapy with post therapy whole body scan showing no evidence of radioavid foci aside from the thyroid bed.

The malignant potential of cystic nodule(s) should never be overlooked in certain select patients even if it carries a low chance of malignancy. The diagnosis of PTC relies primarily on the typical nuclear features, however in cases of histologic uncertainty, immunohistochemical stains such as HBME-1 may be used to help classify unusual presentations of PTC. Treatment and monitoring of Cystic Papillary Thyroid Carcinoma follows the conventional guideline on solid PTC.
\end{abstract}

Key words: cystic papillary thyroid carcinoma, HBME-1, papillary thyroid carcinoma

\section{INTRODUCTION}

Thyroid nodules are a common endocrine problem encountered worldwide. They are defined as lesions arising from thyroid gland that is radiologically separate from the surrounding parenchyma. ${ }^{1}$ Clinically, thyroid nodules greater than 1 centimeter are usually palpable while those smaller can only be seen using an ultrasound. The prevalence of palpable thyroid nodules is approximately $5 \%$ in women and $1 \%$ in men in iodine-sufficient areas. In contrast, the incidental findings of thyroid nodule on ultrasound can be as high as $19-68 \%$ with a preponderance towards elderly females. ${ }^{1}$ In the Philippines, the most recent prevalence of goiter is estimated to be at $8.9 \%$ with $56 \%$ having diffuse enlargement and $44 \%$ having nodular goiters. ${ }^{2}$

The importance of clinical work up for asymptomatic thyroid nodule is to determine whether the lesion is benign or malignant, as management for both largely differs. The most common well differentiated malignant thyroid nodules are Papillary and Follicular thyroid carcinomas with PTC accounting for about $80 \%$ of cases. ${ }^{1,3}$ In a local study done by Lo et al., majority of the well differentiated thyroid cancers were PTC with alarmingly aggressive characteristics. ${ }^{4}$ Certain ultrasound characteristics can help differentiate benign from malignant thyroid nodules. The finding of a purely cystic nodule has a less than $1 \%$ chance of malignancy and biopsy may not even be warranted. ${ }^{1}$

We report a case of a PTC presenting with a purely cystic appearance, diagnosed only after surgery. Histopathology showed a suspicious lesion for thyroid malignancy and it was confirmed using immunohistochemical staining.

\section{CASE}

A 45-year-old male initially consulted because of sinusitis with multiple palpable thyroid nodules on examination. He had no hypothyroid or hyperthyroid symptoms or significant radiation exposure to the neck. The family has no history of thyroidal illness but a close relative has colonic cancer. Baseline ultrasound revealed normal thyroid glands with multiple nodules: On the right thyroid was a small $0.58 \mathrm{~cm}$ ovoid solid mass (Figure 1A); and a big $3.08 \times 2.82 \times 2.11 \mathrm{~cm}$ ovoid cystic mass (Figure 1B); while on the left thyroid there was a small $0.21 \mathrm{~cm}$ ovoid cyst (Figure 1C). Thyroid function tests were normal, and patient was started on levothyroxine suppression therapy.

On follow up, the cystic mass decreased in size until 19 months when the ovoid cyst seen on the right thyroid increased in size by about $2 \%$ and now appeared as a welldefined cyst with no vascularity or calcifications. At this time, fine needle aspiration biopsy was done revealing blood and colloid. He underwent right thyroidectomy and the specimens were sent for both frozen and permanent sections. Grossly, the specimen on cut sections showed a cystic cavity measuring $3.2 \mathrm{~cm}$ in diameter and at the upper pole, a note or a reddish-brown nodule measuring $0.5 \mathrm{~cm}$ in greatest dimension.

Microscopically, the reddish nodule is composed of randomly oriented papillae with fibrovascular cores 

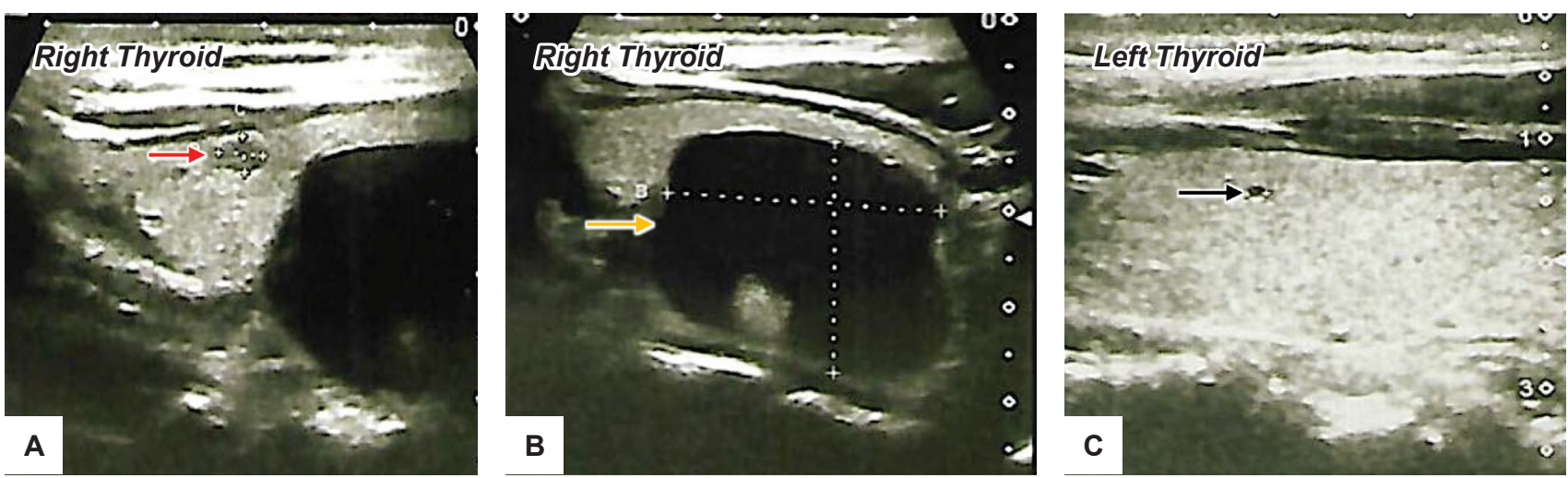

Figure 1. (A) Ultrasound shows a $0.58 \mathrm{~cm}$ solid mass (red arrow); (B) shows a $3.08 \times 2.82 \times 2.11 \mathrm{~cm}$ ovoid cystic mass (yellow arrow) both located at the right thyroid lobe; (C) shows a $0.21 \mathrm{~cm}$ ovoid cyst (black arrow) at left thyroid lobe.
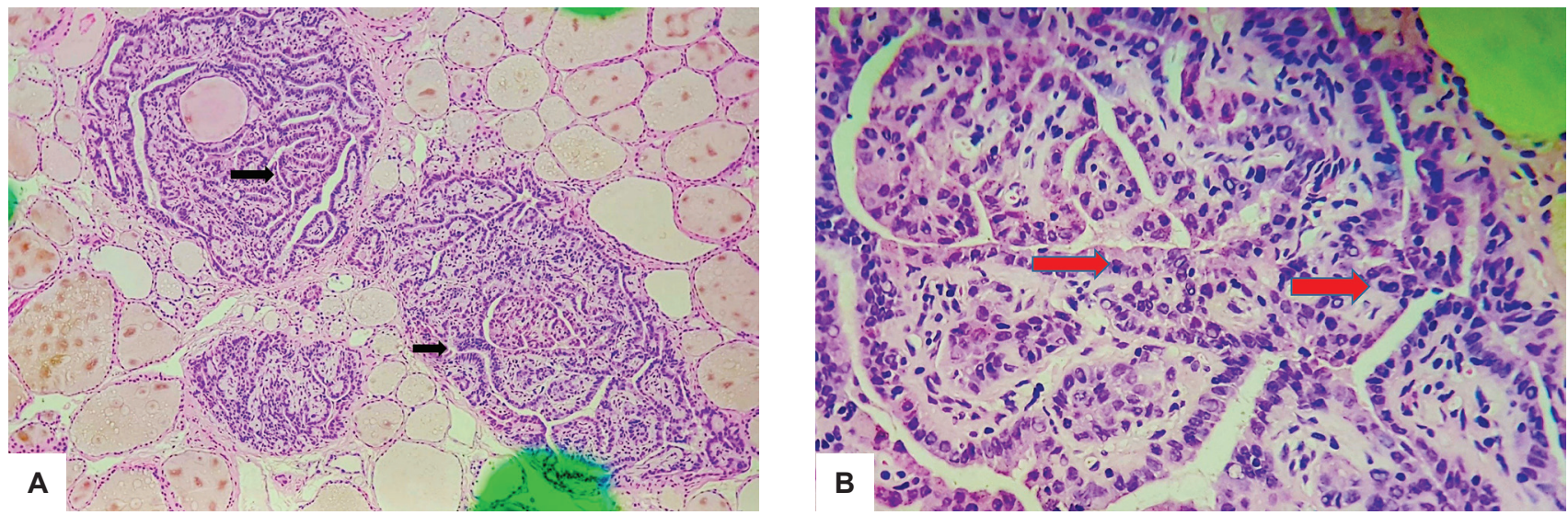

Figure 2. $0.5 \mathrm{~cm}$ solid nodule seen on ultrasound. (A) Black arrows show papillae with fibrovascular cores (H\&E, 40x); (B) Red arrows show cuboidal cells with overlapping nuclei (H\&E, 100x).
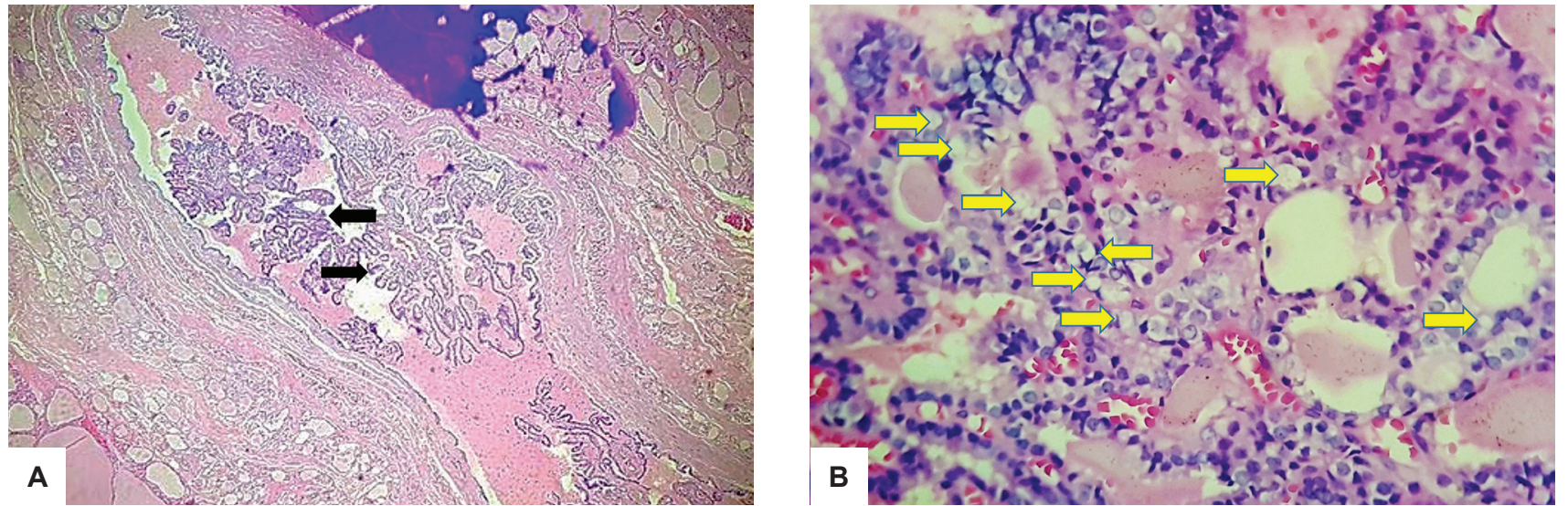

Figure 3. $3.08 \times 2.82 \times 2.11 \mathrm{~cm}$ ovoid cystic mass on ultrasound. (A) Black arrows show papillae formation (H\&E, 40x); (B) On higher magnification (H\&E, 400x), yellow arrows show clear or empty nucleus commonly termed as "Orphan Annie Eye." There is also no extensive infiltration by lymphocytes or macrophages noted and the follicules have abundant colloid material.

(Figure 2A). On higher magnification, the nodule showed papillae lined by cuboidal cells showing round to oval overlapping nuclei with finely dispersed optically clear chromatin, inconspicuous nucleoli and ample cytoplasm. Furthermore, some tumor cells have prominent grooves and occasional pseudoinclusions (Figure 2B).

The cystic cavity, on the other hand, is surrounded by tissues compose of variable sized dilated follicles with flattened to hyperplastic epithelium forming papillae projections into the lumen (Figure 3A). The cells lining the papillary projections were noted to show occasional optical clearing (Figure 3B) thus the pathologist requested for Hector Battifora Mesothelial-1 (HBME-1). The HBME-1 immunostain showed a diffuse membranous staining of the tumor cells (Figure 4).

The patient underwent completion thyroidectomy, and the histopathology results of the left thyroid showed nodular goiter with interstitial fibrosis. Four weeks post 

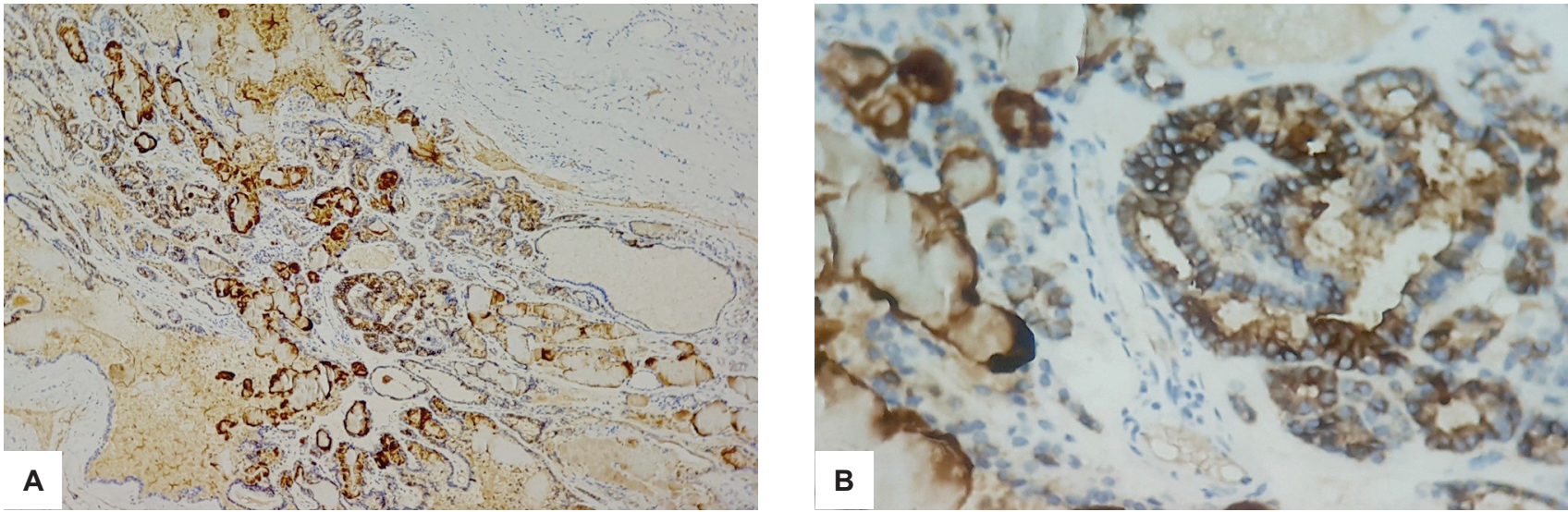

Figure 4. (A) Staining for HBME-1 which appears as a brownish tan stain (HBME-1, 40x); (B) On higher magnification, shows HBME-1 positive seen within the plasma membrane of the tumor cells (HBME-1, 400x)

operation, the patient received high dose radioactive iodine therapy. Whole body scan taken 1 week after radiation therapy revealed no evidence of radioavid foci aside from the thyroid bed and patient was categorized as Stage $2\left(\mathrm{~T}_{2} \mathrm{~N}_{0} \mathrm{M}_{0}\right)$.

\section{DISCUSSION}

Well differentiated thyroid cancers usually appear on ultrasonography as solid or complex solid nodules with irregular margins, microcalcifications, a taller than wider appearance, and with rim calcifications. These features have an estimated risk of up to $90 \%$ for malignancy and prompts fine needle biopsy for lesions 1 centimeter and above. ${ }^{1}$ In this patient, the ultrasound findings were not suggestive of any thyroid malignancy and the indication for fine needle biopsy was to decrease the size of the lesion.

A true thyroid cyst composed of a liquid center lined by cells is rare and most clinically palpated thyroid cysts are solid nodules that have undergone cystic degeneration. These cysts are commonly benign thyroid adenomas. ${ }^{5}$ The risk of malignancy in cystic nodules is about $5-10 \%$ and it increases as the proportion of the solid part of nodule increases. ${ }^{5}$ In a study done by de los Santos et al., it was noted that malignant cystic nodules were Papillary thyroid carcinoma on histopath. ${ }^{6}$ Furthermore, it was noted that cystic lesions are as likely as solid lesions to harbor malignancy and this distinction cannot be accurately predicated from the clinical characteristics of the cysts nor the patient's demographic data. ${ }^{6}$ As a recommendation, most cysts that are not abolished by aspiration should be excised.

According to the World Health Organization's Classification of Tumors, ${ }^{7}$ the histology of PTC is sufficient for its diagnosis and consists of nuclear features showing an enlarged, oval shaped, elongated and overlapping appearance. The nuclei may also show optical clearing or a ground glass appearance. Irregularity of nuclear contours including grooves and nuclear pseudoinclusions are likewise common. Furthermore, in tumors that lack the complexes of papillary structures, the diagnosis of PTC relies heavily on the aforementioned features, which should be present in significant proportions of the neoplasm. ${ }^{7}$ The typical papillary architecture of PTC consists of papillae that are complex with branches, and some may appear edematous. ${ }^{7}$ The papillae are covered by epithelium with disturbed polarity and pale or eosinophilic cytoplasm. ${ }^{7}$ For our case, the solid $0.5 \mathrm{~cm}$ undoubtedly was PTC based on both the architectural and nuclear features. However, for the cystic cavity even if it has the typical epithelium of papillae projections, the nucleus only showed occasional optical clearing. The presence of optical clearing although very common in PTC, is not pathognomonic of the tumor, ${ }^{8}$ and thus immunohistochemistry was utilized.

PTC are usually reactive to immunohistochemical stains like cytokeratins, thyroglobulin and thyroid transcription factor-1 (TTF-1) and are negative for synaptophysin and chromogranin. ${ }^{7}$ Galectin-3 and Hector Battifora Mesothelial-1 (HBME-1) are also expressed in high proportions but according to the WHO consensus, it is not specific for PTC. ${ }^{7}$ HBME-1 is an unelucidated membrane antigen found in the microvilli of mesothelial cells, normal tracheal epithelium, and adenocarcinomas of the lung, pancreas and breast. ${ }^{9}$ No expression is seen in normal thyroid tissues and when present, suggests malignant lesions that could either be PTC, Follicular Variant of PTC (FVPTC), Follicular Thyroid Carcinoma (FTC), Poorly Differentiated Thyroid Carcinoma (PDC), Undifferentiated Thyroid Carcinoma (UDC) and Hurthle Cell Carcinoma (HCC). FVPTC on histology are composed of small to medium sized, irregularly shaped follicles with virtually no papillary structures. ${ }^{7}$ They have variable amount of colloid that appear hypereosinophilic and scalloped and the majority of cells lining the follicles contain large clear nuclei with grooves and nuclear pseudoinclusions that are similar to the classic PTC. ${ }^{7}$ However, PTC remains overall to be the most consistent thyroid malignancy that expresses HBME-1. ${ }^{9}$

In a recent study that looked into the clinically utility of HBME-1 for the diagnosis of PTC, it had a sensitivity and specificity of $87 \%$ and $96 \%$ respectively. ${ }^{10}$ Compared to Cytokeratin-19 (CK19), HBME-1 was specific at 96\% but CK19 appears more sensitive at 96\%. ${ }^{10}$ Furthermore, 100\% specificity is seen with coexpression of HBME-1/CK19. ${ }^{10}$

According to the WHO consensus, PTC is positive for HBME-1 when it is present primarily within the plasma membrane of the tumor cells. ${ }^{7}$ Other authors define 
positivity particularly as diffuse membranous positivity. ${ }^{10}$ As noted earlier, FVPTC similarly express HBME-1 but since they lack the typical papillary structures, the stain appears within the cell membrane of the follicles. ${ }^{11}$ In contrast, no staining is seen for nodular hyperplasia. ${ }^{11} \mathrm{Nasr}$ et al., looked into the immunohistochemical markers for the diagnosis of PTC, and HBME-1 showed high sensitivity and specificity, although it can similarly be present in focal areas of Hashimoto's Thyroiditis. ${ }^{12}$ Since nuclear features of Hashimoto's thyroiditis may overlap with PTC including nuclear clearing, the positivity of HBME-1 per se should not be equated with the diagnosis of PTC without considering its histology. ${ }^{12}$

For our case, HBME-1 was primarily requested for the cystic lesion because of the presence of optical clearing that suggested a malignant lesion. Ideally, other stains such as CK19 and Galectin-3 should have been requested together with HBME-1 to improve specificity. However, since the cystic lesion stained positive for HBME-1 but lacked the pathognomonic lymphocytic infiltration seen in Hashimoto's Thyroiditis, it was eventually diagnosed to be classic PTC appearing in a cystic lesion. Moreover, because the architectural characteristic of the cystic lesions had papillae, classic PTC was favored more than FVPTC.

There have been several case reports published on Cystic Papillary Thyroid Carcinoma including those by Patil ${ }^{13}$ Baser ${ }^{14}$ and Pratinidhi. ${ }^{15}$ In those case reports, the patients were all females presenting with large multiple neck masses that were noted on examination. Majority of the ultrasonography findings were cystic with solid components. In contrast, our patient was a male that presented only with an incidental finding of a palpable neck mass. The nodule was noted on ultrasound to be ovoid and purely cystic. Unfortunately, however no pattern can be deduced on patient characteristics that are highly suggestive of Cystic Papillary Thyroid Carcinoma (CPTC) and the American Thyroid Association strongly recommends surgical intervention for cystic lesions greater than $4 \mathrm{~cm} .{ }^{1}$ In terms of histopathology, no nuclear or architectural characteristic feature can be seen as pathognomonic for CPTC and in the published case reports, the histology appears as classic PTC with prominent cystic degenerative changes. Furthermore, majority of the case reports on CPTC did not utilize immunohistochemical staining and was diagnosed solely on nuclear features. In the case report of Rahamat et al., that described two cases of male patients that have PTC presenting as a lateral neck cyst, the immunohistochemical stains used were TTF-1, thyroglobulin (TGB) and Cytokeratin (CK19). ${ }^{16}$ TTF-1, a nuclear protein composed of a single polypeptide of 371 amino acid belongs to the family of homeodomain transcription factors and is the most commonly used immunomarker to identify thyroid or lung primary tumor in the setting of metastasis. ${ }^{9}$ Thyroglobulin is a thyroid hormone precursor that is synthesized by the thyrocytes, transported to the apical surface and secreted into the follicles and constitute the major component of colloid. ${ }^{9}$ Thyroglobulin and TTF-1 are commonly used as a marker of thyroid organ determination and for that case, it was used to help differentiate a primary from a metastatic malignancy. Like HBME-1, CK19 is an immunohistochemical stain used for the differential diagnosis of thyroid gland neoplasm. It is a low- molecular-weight cytokeratin found in a variety of simple or glandular epithelial seen in both normal and neoplastic epithelium. ${ }^{9}$ In some studies, CK19 staining was seen lacking specificity for PTC or malignancy. ${ }^{9}$ It is reported to have $82.2 \%$ sensitivity for PTC and $44.3 \%$ for FTC while it has an overall specificity for thyroid malignancy at $63.1 \% .{ }^{9}$ Overexpression of CK19 is a good indicator for PTC but should be part of the panel of immunomarkers in the diagnosis of PTC. ${ }^{9}$

Management for this patient's case followed the recommended guideline for well differentiated thyroid carcinoma, including total thyroidectomy followed by post-operative radioactive iodine therapy and lifetime levothyroxine suppression therapy.

\section{CONCLUSION}

The chance of malignancy of purely cystic nodule is minimal and estimated at $<1 \%$ while those with a partially cystic nodule without suspicious features is $<3 \%$. Yet, its malignant potential should not be overlooked because thyroid cancers can occasionally present as cystic nodules. Diagnosis of PTC should be made by histology but in case of uncertainty or an unusual appearance, immunohistochemical stains such as HBME-1 are useful tests to help differentiate benign from malignant lesions. The results should be interpreted in the context of the histologic appearance especially if the stain used is not $100 \%$ specific. Combination with other stains may also be necessary to improve accuracy. The treatment of CPTC is the same as its solid counterpart and follows an indolent course.

\section{Ethical Consideration}

Patient consent was obtained before submission of the manuscript.

\section{Statement of Authorship}

All certified fulfillment of ICMJE authorship criteria.

\section{Author Disclosure}

The authors declared no conflict of interest.

\section{Funding Source}

None.

\section{References}

1. Haugen BR, Alexander EK, Bible KC et al. 2015 American Thyroid Association management guidelines for adult patients with thyroid nodules and differentiated thyroid cancer. Thyroid. 2016;26(1):1133. PMID: 26462967. PMCID: PMC4739132.https://doi.org/ 10.1089/ thy.2015.0020.

2. Raboca-Carlos J, Kho SA, et al. The Philippine Thyroid Disease Study (PhilTiDes1): Prevalance of thyroid disorders among adults in the Philippines. J ASEAN Fed of Endocr Soc. 2012;27(1): 27-33. https://doi. org/10.15605/jafes.027.01.04.

3. Hundahl SA, Fleming ID, Fremgen AM, Menck HR. A National Cancer data base report on 53, 856 cases of thyroid carcinoma treated in the US from 1985-1995. Cancer. 1998;83(12):2638-48. PMID: 9874472. https://doi.org/10.1002/(sici)1097-0142(19981215)83:12<2638::aidcncr31>3.0.co;2-1.

4. Lo TE, Uy AT, Maningat PD. Well-differentiated thyroid cancer: The Philippine General Hospital experience. Endocrinol Metab (Seoul). 2016;31(1):72-9. PMID: 26754584. PMCID: PMC4803565. https://doi org/10.3803/EnM.2016.31.1.72.

5. Ross D. Cystic thyroid nodules. UpToDate. https://www.uptodate. com/contents/cystic-thyroid-nodules.

6. de los Santos ET, Keyhani-Rofagha S, Cunningham JJ, Mazzaferri EL Cystic thyroid nodules. The dilemma of malignant lesions. Arch Intern Med. 1990;150(7):1422-7. PMID: 2196027. https://doi.org/10.1001/ archinte.150.7.1422. 
7. Pathology and genetics of tumors of the endocrine organs, 3rd ed. WHO classification of tumours, vol. 8, DeLellis RA, Lloyd RV, Heitz PU, ed.; 2004.

8. Hapke MR, Dehner LP, The optically clear nucleus: A reliable sign of papillary thyroid carcinoma of the thyroid. Am J Surg Pathol. 1979;31(1):31-8. PMID: 534382. https://doi.org/10.1097/00000478197902000-00004.

9. Liu $\mathrm{H}$ and Lin F. Application of immunohistochemistry in thyroid pathology. Arch Pathol Lab Med. 2015;139(1):67-82. PMID: 25549145. https://doi.org/10.5858/arpa.2014-0056-RA.

10. Scognamiglio T, Hyjek E, Kao J, Chen YT. Diagnostic usefulness of HBME-1, galectin-3, CK19, and CITED1 and evaluation of their expression in encapsulated lesions with questionable features of papillary thyroid carcinoma. Am J Clin Pathol. 2006;126(5):700-8. PMID: 17050067. https://doi.org/10.1309/044V-86JN-2W3C-N5YB.

11. Erdogan-Durmus S, Ozcan D, Yarikkaya E, Kurt A, Arsian A. CD56, HBME-1 and cytokeratin 19 expressions in papillary thyroid carcinoma and nodular thyroid lesions. J Res Med Sci. 2016; 21:49. PMID: 27904595. PMCID: PMC5121990. https://doi.org/10.4103/17351995.183986

12. Nasr MR, Mukhopadhyay S, Zhang S, et al. Immunohistochemical markers in diagnosis of Papillary Thyroid Carcinoma: Utility of HBME-1 combined with CK19 immunohistostaining. Mod Pathol. 2006;19(12):1631-7. PMID: 16998461. https://doi.org/10.1038/ modpathol.3800705.
13. Patil VS, Vijayakumar A, Natikar N. Unusual presentation of cystic papillary thyroid carcinoma. Case Rep Endocrinol. PMID: 23133761. PMCID: PMC3485764. https://doi.org/ 10.1155/2012/732715.

14. Baser B, Munjal VR, Roy MT. Papillary carcinoma of the thyroid with unusual presentation. Indian J Otolaryngol Head Neck Surg. 2015;67(suppl 1):145-8. PMID: 25621272. PMCID: PMC4298624. https:// doi.org/10.1007/s12070-014-0746-y.

15. Pratinidhi S, Panda S, Das D. Papillary thyroid carcinoma presented as cystic neck mass: A rare presentation. Indian Journal of Clinical Practice. 2014; 24(4): 339-41. http://medind.nic.in/iaa/t13/i9/ iaat13i9p339.pdf.

16. Rahmat F, Kumar A, Muthu AKM, Gopal NSR, Han SI, Yahaya AS. Papillary thyroid carcinoma as a lateral neck cyst: A cystic metastatic node versus an ectopic thyroid tissue. Case Rep Endocrinol. 2018;2018:5198297. PMID: 30420925. PMCID: PMC6211211. https://doi. org/10.1155/2018/5198297.

Authors are required to accomplish, sign and submit scanned copies of the JAFES Author Form consisting of: (1) Authorship Certification, that authors contributed substantially to the work, that the manuscript has been read and approved by all authors, and that the requirements for authorship have been met by each author; (2) the Author Declaration, that the article represents original material that is not being considered for publication or has not been published or accepted for publication elsewhere, that the article does not infringe or violate any copvrights or intellectual property rights, and that no references have been made to predatory/ suspected predatory journals; (3) the Author Contribution Disclosure, which lists the specific contributions of authors; and (4) the Author Publishing Agreement which retains author copyright, grants publishing and distribution rights to JAFES, and allows JAFES to apply and enforce an Attribution-Non-Commercial Creative Commons user license. Authors are also required to accomplish, sign, and submit the signed ICMJE form for Disclosure of Potential Conflicts of Interest. For original articles, authors are required to submit a scanned copy of the Ethics Review Approval of their research as well as registration in trial registries as appropriate. For manuscripts reporting data from studies involving animals, authors are required to submit a scanned copy of the Institutional Animal Care and Use Committee approval. For Case Reports or Series, and Images in Endocrinology, consent forms, are required for the publication of information about patients; otherwise, appropriate ethical clearance has been obtained from the institutional review board. Articles and any other material published in the JAFES represent the work of the author(s) and should not be construed to reflect the opinions of the Editors or the Publisher.

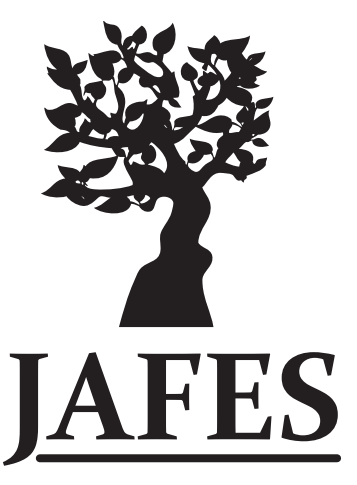

\section{Send your paper to the publication pathway. Instructions to Authors at www.ASEAN-endocrinejournal.org.}

